\title{
Coarse wavelength division (de)multiplexer using interleaved angled multimode interferometer structure
}

\author{
Y. Hu, ${ }^{a)}$ F. Y. Gardes, D. J. Thomson, G. Z. Mashanovich, and G. T. Reed \\ Optoelectronics Research Centre, University of Southampton, Southampton, SO17 1BJ, UK
}

We have demonstrated a new coarse wavelength (de)multiplexing CWDM structure on the silicon-on-insulator (SOI) platform. It is composed of two 4-channel angled multimode interferometers (AMMIs) interleaved with an imbalanced Mach-Zehnder interferometer (MZI) leading to an 8-channel device. The device requires only single lithography and etching steps for fabrication and has good tolerance to fabrication errors in terms of waveguide width. The insertion loss and cross talk achieved are $3-4 \mathrm{~dB}$ and $-(15-20) \mathrm{dB}$, respectively. Potential is shown for achieving improved performance using larger waveguide bending radii in the MZI arms and/or (a) local heater(s) for refractive index tuning.

a) Electronic mail: y.hu@soton.ac.uk 
The commercialisation of silicon photonics integrated circuits requires low-cost wavelength division (de)multiplexers (WDMs) with low insertion loss, low cross talk and relaxed fabrication tolerance to be integrated with other silicon photonics components on the same waveguide platform, e.g. the silicon-on-insulator (SOI) platform. Recently, the WDM technology based on non-silicon based platforms with low refractive index contrast, e.g. silica-on-silicon, GaAs and InP, has been successfully adapted to the SOI platform which has a large refractive index contrast. However, extra complexity is usually required in SOI based WDMs for improved spectral responses. For example, in SOI based arrayed waveguide gratings (AWGs) and planar concave gratings (PCGs), an extra lithography and etching step is required at the interface between input/output waveguides and the free propagation region (FGP) to reduce the insertion loss and crosstalk ${ }^{1,2}$; in ring/race-track resonators, a $2 \times 2$ multimode interferometer (MMI) was used in the coupler section ${ }^{3}$ for wavelength independent operation. In addition, the spectral responses of those SOI based WDMs are very sensitive to fabrication errors in waveguide dimensions, with a sensitivity of spectral shift to waveguide dimension error on the order of 100 $\mathrm{GHz} / \mathrm{nm}^{4}$. Previously, we demonstrated an angled multimode interferometer (AMMI) structure as a 4-channel coarse wavelength division multiplexer (CWDM) on the SOI platform ${ }^{5}$. It has the distinct advantage of low insertion loss $(<2 \mathrm{~dB})$ and requires only a single lithography and etching step for fabrication. However, the design for a single AMMI with more channels $(>8)$ and narrower channel spacing $(<5 \mathrm{~nm})$ on the SOI platform results in a device length in the centimetre scale. Its spectral response also deteriorates with additional channels. In this letter, we show an interleaved AMMI CWDM structure with an increased number of channels and reduced channel spacing whilst low insertion loss and low crosstalk are maintained and the device length is not significantly increased. An 8-channel WDM device comprising two AMMIs and one 
imbalanced Mach-Zehnder interferometer (MZI) has been demonstrated experimentally. Its fabrication error sensitivity has also been studied.

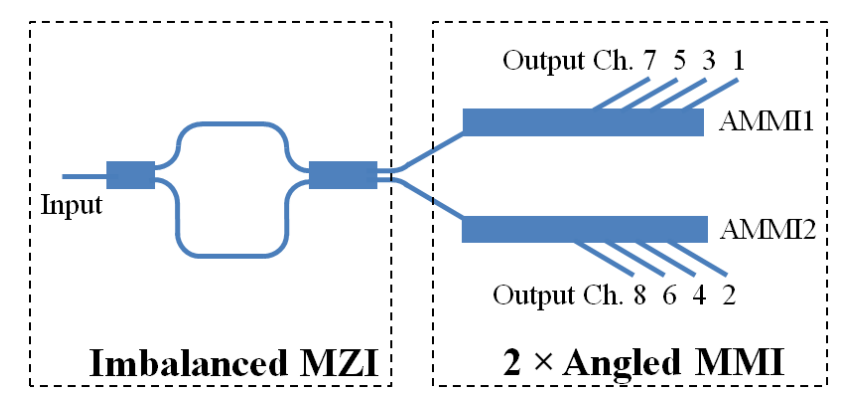

Fig. 1. (Color online) Design of the 8-channel interleaved AMMI.

The design concept of the 8-channel interleaved AMMI is illustrated schematically in Fig. 1. The device is composed of two parts, an imbalanced Mach-Zehnder interferometer (MZI) and two 4-channel AMMIs. The AMMI sections of the integrated device could be folded back to be positioned alongside the MZI so that the overall device length is not significantly increased. Clearly the overall footprint increases a little due to the extra MZI, but the device is primarily limited by a single AMMI's length. The free spectral range (FSR) of the MZI, $\Delta \lambda_{M Z I}$, and the channel spacing of the AMMI, $\Delta \lambda_{\text {AMMI }}$, satisfy the condition:

$$
\Delta \lambda_{A M M I}=\Delta \lambda_{M Z I}
$$

The peak transmission wavelengths $\left(\lambda_{1}-\lambda_{8}\right)$ of the 8 output channels in the AMMIs are determined by the self-imaging condition of the AMMI

$$
\lambda_{i}=\frac{4 n_{e f f, A M M I} \times W_{A M M I}^{2}}{L_{i}} \quad(i=1, \cdots, 8)
$$

where, $n_{\text {eff,AMMI }}$ is the effective refractive index of the fundamental mode in the AMMI's multimode waveguide, $W_{A M M I}$ is the width of the multimode waveguide, and $L_{i}$ is the axial length 
between the input and the $i$ th output channel. These wavelengths should satisfy the interleaving condition:

$$
\lambda_{2 n}=\lambda_{2 n-1}+\frac{\Delta \lambda_{M Z I}}{2} \quad(i=1,2,3,4)
$$

In addition, the peak resonant wavelengths of the MZI and AMMI have to match each other, i.e.:

$$
\lambda_{i}=\frac{2 n_{g, M Z I} \times \Delta L_{M Z I}}{m+i} \quad(i=1, \cdots, 8)
$$

where, $m$ is an integer, $n_{g, M Z I}$ is the average group index of the MZI arm waveguides at wavelengths from $\lambda_{1}$ to $\lambda_{8}$, and $\Delta L_{M Z I}$ is the arm length difference of the imbalanced MZI. Therefore, the whole structure is arranged in a way that the interleaved resonant wavelengths of the MZI passing through the upper and lower MZI output waveguides are distributed respectively to the 8 output channels in the two 4-channel AMMIs, and the device has a channel spacing of $\Delta \lambda_{A M M I} / 2$ or $\Delta \lambda_{M Z I} / 2$. Equations (1)-(4) give the design rules of the interleaved AMMI.

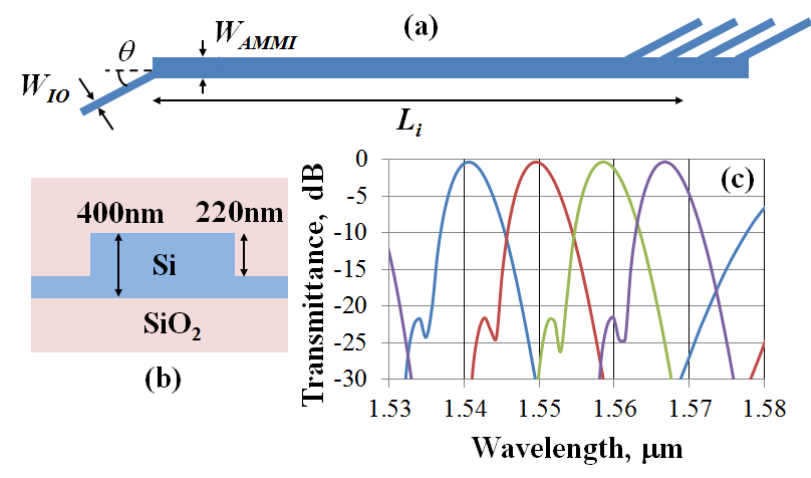

Fig. 2. (Color online) (a) Design layout, (b) cross section, and (c) simulated spectral response of AMMI1. 
Our design starts with the optimisation of the two 4-channel AMMIs' spectral responses. The design and simulation results are shown in Fig. 2. Using the same waveguide platform and optimisation method demonstrated previously ${ }^{5}$, we have achieved a simulated spectrum for AMMI1, which is shown in Fig. 2(c). Compared with our previous work, improved spectral response was shown. The channel spacing of a single 4-channel AMMI has been reduced from $20 \mathrm{~nm}$ to $9 \mathrm{~nm}$ so that the wavelength span of an 8-channel interleaved AMMI is less than the bandwidth of a typical Erbium Doped Fibre Amplifier (EDFA) at about $35 \mathrm{~nm}$. This was achieved by increasing the multimode waveguide width, $W_{\text {AMMI }}$, from $12 \mu \mathrm{m}$ to $25 \mu \mathrm{m}$ at the cost of an increase of device length from $1.2 \mathrm{~mm}$ to $4.7 \mathrm{~mm}$. The input/output waveguide width, $W_{I O}$, increased from $5 \mu \mathrm{m}$ to $9 \mu \mathrm{m}$ and the tilted angle, $\theta$, was optimised to be $17.3^{\circ}$, so that the insertion loss was further reduced from $\sim 2 \mathrm{~dB}$ to $<1 \mathrm{~dB}$ and the cross talk from $-18 \mathrm{~dB}$ to $-25 \mathrm{~dB}$. A commercial software package, FIMMWAVE, was used for selecting those structural parameters for optimised spectral response. The structural parameters of the optimised design are shown in Table I. Further reduction of channel spacing is possible, however, the device length would increase to centimetre scale if a channel spacing of $<5 \mathrm{~nm}$ is required whilst the insertion loss and the cross talk would be maintained at the same level.

TABLE I. Designed structural parameters for the two 4-channel AMMIs

\begin{tabular}{|c|c|c|c|c|}
\hline \multicolumn{5}{|c|}{$W_{I O}=9 \mu \mathrm{m}, \theta=17.3^{\circ}, W_{\text {AMMI }}=25 \mu \mathrm{m}$} \\
\hline \multirow{3}{*}{ AMMI1 } & $L_{1}(\mu \mathrm{m})$ & $L_{3}(\mu \mathrm{m})$ & $L_{5}(\mu \mathrm{m})$ & $L_{7}(\mu \mathrm{m})$ \\
& & & & 4600 \\
\cline { 2 - 5 } & 4702 & 4668 & 4634 & $L_{8}(\mu \mathrm{m})$ \\
\hline AMMI2 & $L_{2}(\mu \mathrm{m})$ & $L_{4}(\mu \mathrm{m})$ & $L_{6}(\mu \mathrm{m})$ & \\
\hline
\end{tabular}




\begin{tabular}{|l|l|l|l|l|}
\hline & 4685 & 4651 & 4617 & 4584 \\
\hline
\end{tabular}

Once the design parameters of the AMMIs are fixed, we were able to design the matching MZI for the interleaved device using equation (4) and the structural parameters of the SOI platform shown in Fig. 2(b), which resulted in a singlemode MZI arm waveguide width of 450 $\mathrm{nm}$ and an arm length difference of $\Delta L_{M Z I}=68 \mu \mathrm{m}$. For low insertion loss and wavelength independent operation, an optimised $1 \times 2$ MMI power splitter and a $2 \times 2$ MMI coupler with simulated insertion loss of about $0.1 \mathrm{~dB}$ for both were used in the MZI. The two output branches from the MZI were connected to the inputs of AMMI1 and AMMI2 via adiabatic tapers so that the light from the MZI was maintained in the waveguides' fundamental modes when it propagated to the $9 \mu \mathrm{m}$-wide input waveguides of the AMMIs.

In consideration of effective coupling and normalization of light for insertion loss measurement, we designed a taper-grating unit to be incorporated into the design. This unit is composed of a $450 \mathrm{~nm}$-wide singlemode waveguide in connection with an adiabatic taper up to a $10 \mu \mathrm{m}$-wide waveguide, where surface gratings were fabricated for in/out-coupling of light through optical fibers. The input waveguide from the MZI is directly connected to one of the taper-grating units. The eight $9 \mu \mathrm{m}$-wide output waveguides from the AMMIs were first connected to $450 \mathrm{~nm}$-wide singlemode waveguides via adiabatic tapers which act as mode filters, and then to eight taper-grating units. A separate structure incorporating two of the taper-grating units connected back-to-back was used for normalization. 


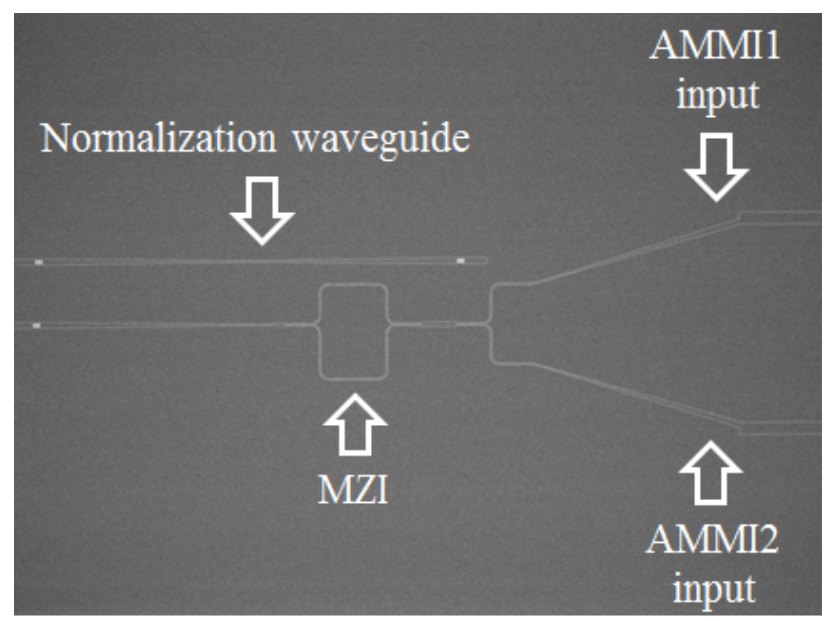

(a)

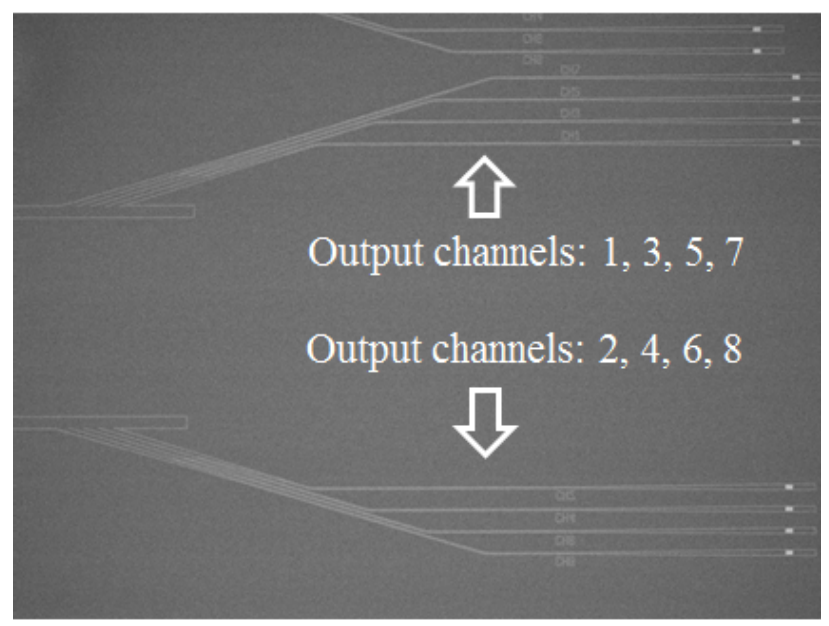

(b)

Fig. 3. (Color online) Scanning electron microscope (SEM) images of the fabricated interleaved AMMI. (a) The section including the normalization waveguide, the MZI and the AMMIs' inputs; (b) The section of output channels.

The designed devices including the stand-alone imbalanced MZI, AMMIs and the integrated interleaved AMMI devices were patterned on a high resolution positive electron beam (e-beam) resist, ZEON ZEP520A, spun on a 6-inch SOI wafer with a silicon overlayer thickness of 400 $\mathrm{nm}$ and buried oxide (BOX) thickness of $2 \mu \mathrm{m}$, The wafer was then written by an e-beam lithography system, JEOL JBX-9300FS. For this particular work, this system produced a stable e-beam 
spot size of $50 \mathrm{~nm}$ and operated at an acceleration voltage of $100 \mathrm{kV}$. It was patterning the wafer in stitching-error-free high-resolution mode within an area of $1 \mathrm{~mm} \times 1 \mathrm{~mm}$. When writing a large pattern, it achieved a stitching error of less than $20 \mathrm{~nm}$ and a positional accuracy of $1 \mathrm{~nm}$. After a single e-beam writing step and resist development, the pattern was transferred to the wafer using inductively coupled plasma (ICP) etching. Finally, a $1 \mu \mathrm{m}$-thick $\mathrm{SiO}_{2}$ protecting layer was deposited on top of the whole wafer using a plasma-enhanced chemical vapor deposition (PECVD) system before it was diced into chips for testing. The scanning electron microscope (SEM) images of the fabricated interleaved AMMI are shown in Fig. 3.

(a)

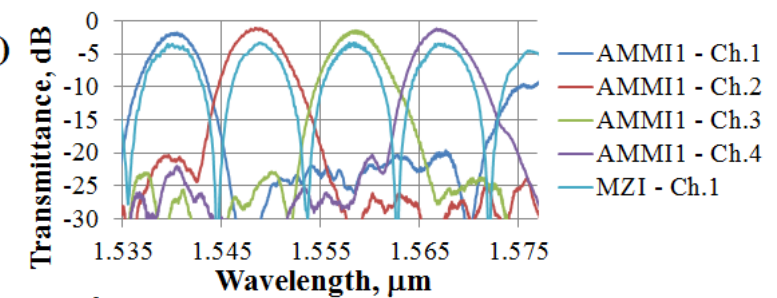

(b)
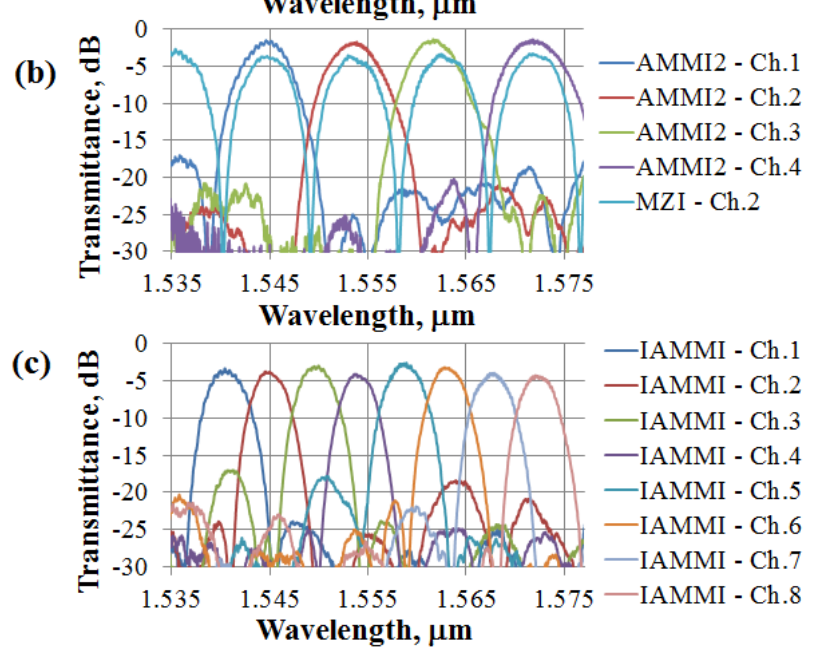

Fig. 4. (Color online) Spectral responses of fabricated devices: (a) 4-channel AMMI1 and MZI channel 1, (b) 4-channel AMMI2 and MZI channel 2, and (c) 8-channel interleaved AMMI (IAMMI).

Fig. 4 shows the measured spectral responses of fabricated devices. As predicted from the simulation, the channel spacing for both the 4-channel AMMI and the MZI is $9 \mathrm{~nm}$. The single AMMI has an insertion loss of 1-1.5 dB and a cross talk of -(18-25) dB across the 4 channels. 
The MZI's insertion loss is slightly higher $(3 \mathrm{~dB})$ as a result of the loss contribution from the $1 \times 1$ MMI power splitter, the $2 \times 2$ MMI coupler, waveguide losses, and waveguide bending losses. Light leakage through the $180 \mathrm{~nm}$-thick slab of the rib waveguide at the bends could account for a large proportion of the insertion loss, and it is expected to be reduced by increasing bending radii. The mismatch of peak wavelengths between the two 4-channel AMMIs and MZI is within $\pm 0.5 \mathrm{~nm}$. As a result, the whole interleaved AMMI has a channel spacing of $4.5 \mathrm{~nm}$, an insertion loss of 3-4 $\mathrm{dB}$ and a cross talk of $-(15-20) \mathrm{dB}$ across the 8 channels. One may notice that the insertion losses of a single stand-alone AMMI and a single stand-alone MZI add up to a value of 4-4.5 dB, which does not match well with that of an interleaved AMMI. This can be due to the uncertainty in waveguide losses and waveguide bending losses of the fabricated devices. We expected a better match of insertion losses to be achieved provided that a number of devices with identical design were fabricated and statistical average of those insertion losses were made. Fig. 4(c) also shows the side lobe peaks of channels 2, 3, and 5 coincide with the main peaks of channels 6,1, and 3 respectively in the spectral responses of the interleaved AMMI. A cross talk of $<-20 \mathrm{~dB}$ across the 8 channels is achievable if those side lobes could be suppressed. One possible solution is to have (a) local heater(s) on top of one (both) of the MZI arms so that the resonant wavelengths and/or channel spacing of the MZI can be tuned independent of the AMMIs, but this would introduce additional power consumption. Nevertheless, the relative position of peak transmission wavelengths between the AMMIs and the MZI can be adjusted for optimised side lobe suppression and minimum insertion loss.

Notably, we achieved a close match of peak wavelengths between AMMI's and MZI's spectral responses without any extra tuning mechanism. In order to understand this fact, we have compared the sensitivity of spectral shift with respect to fabrication error in the width of the MZI's arm waveguide given by:

$$
\Delta \lambda_{M Z I}=\left(\frac{\lambda}{n_{g, M Z I}} \times \frac{\Delta n_{g, M Z I}}{\Delta W_{M Z I}}\right) \Delta W_{M Z I}
$$


and that of the AMMI's multimode waveguide given by:

$$
\Delta \lambda_{A M M I}=\left(\frac{4 W_{A M M I}^{2}}{L_{A M M I}} \times \frac{\Delta n_{e f f, A M M I}}{\Delta W_{A M M I}}\right) \Delta W_{A M M I}
$$

where, $L_{A M M I}$ is the average length of $L_{l}-L_{8}$ in the AMMIs, and the derivatives, $\Delta n_{g, M Z I} / \Delta W_{M Z I}$ and $\Delta n_{e f f, A M M I} / \Delta W_{A M M I}$ were simulated by a commercial software package, FIMMWAVE. Fig. 5 shows the simulated results using equations (5) and (6). The AMMI has an almost linear sensitivity of $\Delta \lambda / \Delta W=12 \mathrm{GHz} / \mathrm{nm}$, which is almost one order of magnitude lower than the sensitivity of conventional SOI devices $(100 \mathrm{GHz} / \mathrm{nm})^{4}$. The MZI has an insensitive point $(\Delta \lambda / \Delta W=0)$ at $\Delta W=20 \mathrm{~nm}\left(W_{M Z I}=470 \mathrm{~nm}\right)$. The sensitivity increases as the width decreases and it has the same value as the AMMI at a point of $\Delta W=-40 \mathrm{~nm}\left(W_{M Z I}=410 \mathrm{~nm}\right)$. At a waveguide width error range of $90 \mathrm{~nm}(-80 \mathrm{~nm}<\Delta \mathrm{W}<10 \mathrm{~nm})$, the relative spectral shift between the AMMI and MZI has a maximum value of $1.2 \mathrm{~nm}$, which corresponds to a relative sensitivity of only $1.8 \mathrm{GHz} / \mathrm{nm}$. Despite the low values of sensitivity and relative sensitivity already achieved, one can see that further optimisation work to be done is to design an interleaved AMMI with matching MZI and AMMIs having the same sensitivity to minor changes in waveguide width as well as the same peak transmission wavelengths, i.e. to satisfy $\Delta \lambda_{\text {AмMI }}=\Delta \lambda_{\text {MII }}$ and $\Delta \lambda_{\text {AMMI }} / \Delta W_{A M M I}=\Delta \lambda_{M Z I} / \Delta W_{M Z I}$ at the same time.

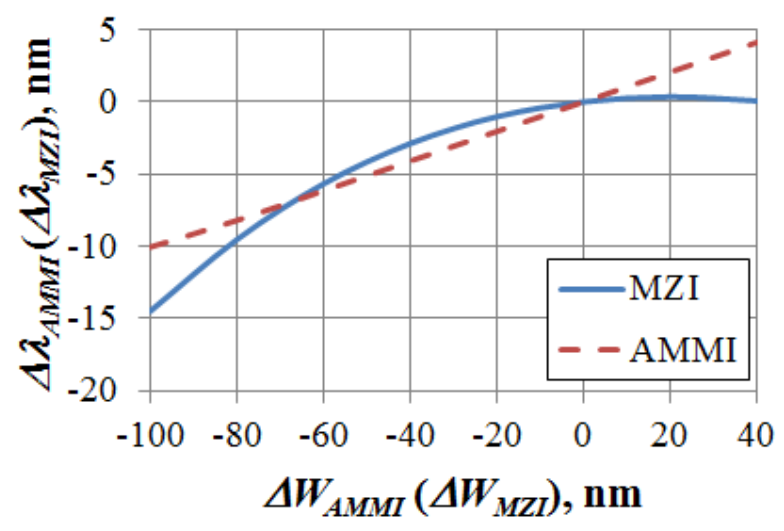


Fig. 5. (Color online) Spectral shift as a function of waveguide width error for the MZI ( $\triangle W_{M Z I}$ - relative to an arm waveguide width of $\left.W_{M Z I}=450 \mathrm{~nm}\right)$ and the AMMI $\left(\Delta W_{A M M I}\right.$ - relative to a multimode waveguide width of $\left.W_{A M M I}=25 \mu \mathrm{m}\right)$.

In conclusion, an 8-channel interleaved angled MMI CWDM structure was demonstrated on the SOI platform. The whole device requires only single-step lithography and etching steps for fabrication and has good tolerance to fabrication errors in terms of the waveguide width. An insertion loss of 3-4 $\mathrm{dB}$ and a cross talk of $-(15-20) \mathrm{dB}$ were achieved. These figures can be further improved by using larger waveguide bending radii in the MZI and/or a heater on the top of MZI $\operatorname{arm}(\mathrm{s})$ for spectral tuning.

\section{Acknowledgement}

The research leading to these results was funded by the UK Engineering and Physical Sciences Research Council (EPSRC) under the grant "UK Silicon Photonics." It has also received funding from the State Key Laboratory of Advanced Optical Communication Systems Networks, China. The fabrication of the (de)multiplexers was carried out at the Southampton Nanofabrication Centre (SNC), University of Southampton, UK.

\section{References}

1. W. Bogaerts, P. Dumon, D. Van Thourhout, D. Taillaert, P. Jaenen, J. Wouters, S. Beckx, V. Wiaux, and R. G. Baets, IEEE J. Sel. Top. Quant. Electron. 12, 1394 (2006). 
2. J. Brouckaert, W. Bogaerts, P. Dumon, D. Van Thourhout, and R. Baets, J. Lightw. Tech. 25, 1269 (2007).

3. D. X. Xu, A. Densmore, P. Waldron, J. Lapointe, E. Post, A. Delâge, S. Janz, P. Cheben, J. H. Schmid and B. Lamontagne, Opt. Exp. 15, 3149 (2007).

4. P. Dong, W. Qian, H. Liang, R. Shafiiha, N. N. Feng, D. Feng, X. Zheng, A. V. Krishnamoorthy, and M. Asghari, Opt. Exp. 18, 9852 (2010).

5. Y. Hu, R. M. Jenkins, F. Y. Gardes, E. D. Finlayson, G. Z. Mashanovich, and G. T. Reed, Opt. Lett. 36, 4488 (2011).

\section{List of figure captions}

Fig. 1. (Color online) Design of the 8-channel interleaved AMMI.

Fig. 2. (Color online) (a) Design layout, (b) cross section, and (c) simulated spectral response of AMMI1.

Fig. 3. (Color online) Scanning electron microscope (SEM) images of the fabricated interleaved AMMI. (a) The section including the normalization waveguide, the MZI and the AMMIs' inputs; (b) The section of output channels.

Fig. 4. (Color online) Spectral responses of fabricated devices: (a) 4-channel AMMI1 and MZI channel 1, (b) 4channel AMMI2 and MZI channel 2, and (c) 8-channel interleaved AMMI (IAMMI).

Fig. 5. (Color online) Spectral shift as a function of waveguide width error for the MZI ( $\Delta W_{M Z I}$ - relative to an arm waveguide width of $\left.W_{M Z I}=450 \mathrm{~nm}\right)$ and the AMMI $\left(\Delta W_{A M M I}\right.$ - relative to a multimode waveguide width of $\left.W_{A M M I}=25 \mu \mathrm{m}\right)$. 